\title{
Potential Candidate Genes for Hypertension with Blood Stasis Syndrome by Microarray and Databases Mining
}

\author{
Lian Yong-Hong, Li Wen-Juan, and Chen Li-Guo
}

\begin{abstract}
Objective: To screen out the potential candidate genes for hypertension with blood stasis syndrome. METHODS: Serum were drawn out from the hypertension patients with blood stasis syndrome (HTBS) and without blood stasis syndrome (HTNS), and the healthy volunteers as control group. HUVEC were cultured with conditioned mediums in which $10 \%$ serum from the different groups for 24 hours. The total RNAs extracted from the HUVEC were labeled with $\mathrm{Cy} 3$ or Cy5 and were hybridized to cDNA microarrays. Gene-array scanner were applied to analyze the gene expressions and the differentially expressed genes were filtered by cy3/cy5 ratios. The functional annotations and enriched pathways of the unique genes for HTBS were analyzed using DAVID. A PPI network was constructed and the potential candidate genes related with blood stasis syndrome were screened out through comparing the degrees of relations with other genes. RESULTS: Based on the network analysis results, 10 potential candidate genes for blood stasis syndrome were screened out and verified through qRT-PCR. Conclusion: Compared with previous methodologies reported in the literatures, the proposed microarray and data mining method has shown a great improvement in analyzing blood stasis syndrome.
\end{abstract}

Index Terms-Blood-stasis syndrome, gene network, hypertension, microarray.

\section{INTRODUCTION}

Traditional Chinese medicine (TCM) is a comprehensive system of medicine characterized by holism and treatment based on syndrome differentiation. "Syndrome" of TCM is a comprehensive generalization of the pathological changes at a certain stage of a disease, including the cause, location, and nature of the disease as well as the trend of development Blood stasis syndrome (BSS), which refers to the pathophysiological state of blood stagnation, delayed blood flow, blockade in blood vessel, and is one of the most common clinical syndromes [1]. Elucidating the mechanisms underlying BSS has long been an important topic in basic and clinical research. However, the primary pathogenic mechanism for BSS remains unclear due to its immense complexity. Thereby, we introduced highthroughput microarrays to identify the different gene profiles of the hypertension patients with BSS. Herein, we further analyzed the molecular function and biologic pathways of the different related genes, and predicted the potential candidate genes for BSS through data mining, which may be a beneficial means for the syndrome study on

Manscript received December 1, 2013; revised February 15, 2014. This work is supported by National Natural Science Foundation of China (No.81173157) and Guangdong Natural Science Foundation of China (No. 10151063201000045).

The authors are with Jinan University, China (e-mail: tchenly@jnu.edu.cn; corresponding author: Chen Li-Guo).
BSS.

\section{MethodS AND MATERIALS}

\section{A. Diagnostic Criteria}

The diagnostic criteria for HT were in accordance with "the Guidelines for prevention and treatment of Hypertension in China" enacted by China's Ministry of Health and Chinese Hypertension League in 2005: systolic pressure $\geq 140 \mathrm{mmHg}$ and/or diastolic pressure $\geq 90 \mathrm{mmHg}$ at rest on at least two different measurements on different days without anti-hypertensive drugs, precluding secondary hypertension. The diagnostic criteria for blood-stasis syndrome were the diagnosis of blood stasis syndrome revised by China professional Committee of Activating Blood and Resolving Stasis in 1986.

\section{B. Patients and Controls}

All 80 patients included in the study were diagnosed with hypertension in Jinan University affiliated hospital between March 2007 and August 2007. They were randomly divided into hypertension patients with blood stasis syndrome (HTBS) $(n=40)$ and hypertension patients without blood stasis syndrome (HTNS) $(n=40)$. The HTBS group consisted of 40 patients (23 male, 17 female) with mean systolic pressure of $168.63 \pm 14.45 \mathrm{mmHg}$, and mean diastolic pressure of $105.93 \pm 8.27 \mathrm{mmHg}$. The HTNS group was comprised of 40 patients ( 25 male, 15 female) with mean systolic pressure of $166.19 \pm 7.56 \mathrm{mmHg}$, and mean diastolic pressure of $103.53 \pm 8.47 \mathrm{mmHg}$. There were no statistical significances between the groups for any of the above parameters $(p<0.05)$. The two groups are essential patients, excluding those with injured target organs, diabetes, or complications in heart, brain, and kidney. We also selected 30 healthy volunteers from Jinan University Medical School as healthy control group, including 17 male and 13 female, with mean systolic pressure of $119.43 \pm 6.47 \mathrm{mmHg}$, and mean diastolic pressure of $79.53 \pm 5.23 \mathrm{mmHg}$. With no significant differences in sex distribution and average age, the subjects in the research are comparable. The research was in accordance with the ethnical standards formulated in the Helsinki Declaration and was approved by the local ethics committee. All individuals were asked to give their written informed consent before participating in the research.

\section{Serum Collection}

Fasting blood samples were taken from the forearms of all the participants, and were placed into dry and sterile, nonanticoagulation vacuum tubes. After the blood samples were self-coagulated, they were centrifuged at $2000 \mathrm{rmp}, 4^{\circ} \mathrm{C}$ for $15 \mathrm{~min}$. And then the supernatant was transposed into a 
sterilized EP tube, stored in $-20^{\circ} \mathrm{C}$ refrigerator until further use for cell culture.

\section{Cell Culture}

Human umbilical vein endothelial cells (HUVEC-C, China Center for type culture collection, Wuhan, Hubei Province, China) were cultured in $25 \mathrm{~cm}^{2}$ culture flasks (Corning). When the cells were in logarithmic growth phase, they were incubated in serum-free $\mathrm{F} 12 \mathrm{~K}$ at $37^{\circ} \mathrm{C}, 5 \% \mathrm{CO}_{2}$ for 24 hours. Then the supernatant was removed and each of the three culture flasks was washed three times with phosphate-buffered saline (PBS). The synchronized cells were cultured in different conditioned mediums (A: 10\% HT with blood-stasis-syndrome serum $+90 \%$ F12K, B: $10 \% \mathrm{HT}$ non-stasis serum $+90 \%$ F12K, C: $10 \%$ norm serum $+90 \%$ $\mathrm{F} 12 \mathrm{~K}$ ) in a humid incubator at $37^{\circ} \mathrm{C}, 5 \% \mathrm{CO}_{2}$ for another 24 hours.

\section{E. RNA Extraction}

When the cells were harvested, the TRIzol reagent (Invitrogen, Carlsbad, CA, USA) was used to extract total RNA according to the manufacturer's protocol. The integrity, quality and purity of total RNA were assessed by an Agilent 2100 Bioanalyzer (Agilent Technologies, CA, USA) and UV spectrometer DU520 (Beckman Coulter, USA). RIN values were ascertained using an Agilent RNA 6000 Nano assay and pass criteria for the RIN value was established at $\geqslant 6$ indicating acceptable RNA integrity. Pass criteria for absorbance ratios were established at A260/A280 $\geqslant 1.8$ and $\mathrm{A} 260 / \mathrm{A} 230 \geqslant 1.5$ indicating acceptable RNA purity. And the passed RNA samples were stored at $-80^{\circ} \mathrm{C}$ until analysis.

\section{F. Microarray Spotting}

Total RNAs of 110 participants (40 HT patients with BSS, 40 HT patients without BSS and 5 healthy volunteers) were used for mRNA expression profiling by the CSE-GE-80 microarray (Chipscreen Biosciences, Shenzhen, China). The microarray contained a total of 8064 gene spots, including 7488 randomly selected cDNA clones, most of which were from IMAGE Consortium cDNA clone collection. In addition, 53 control genes were also included, and each control gene was spotted for 12 times on the chip to ensure the reliability and to test the repeatability of the experimental results. All the samples were spotted into two microarrays using Gen III Microarray Arrayspotter (Amersham pharmacia, Sweden). The microarrays were further treated with ultraviolet cross linking at 400mj (Viber Lourmat, France) for $30 \mathrm{~min}$,

\section{G. Probe Labeling and Microarray Hybridization}

The total RNA samples of the HTBS or the HTNS group and corresponding normal reference were labeled by Cy5$\mathrm{dCTP}$ and Cy3-dCTP respectively in a reverse transcription reaction. The Cy-labeled cDNA samples were purified with QIAquick PCR purification kit and dried by speedvac. After the microarrays were pre-hybridized with hybridization solution at $42^{\circ} \mathrm{C}$ for 6 hours, the fluorescent probes were mixed and denatured at $95^{\circ} \mathrm{C}$ for $5 \mathrm{~min}$, and hybridized into the pretreated microarray with hybridization buffer in a humidified chamber at $42^{\circ} \mathrm{C}$ for 16 hours. Non-specific binding targets were washed away after hybridization, and the microarrays were dried by centrifugation, and scanned with a Generation III array scanner (Amersham Pharmacia, Sweden).

\section{H. Data Normalization and Microarray Analysis}

The scanned images were transferred to digital data with Arrayvision 6.0 (Imaging Research, USA). The raw signal data was normalized with Lowess Regression method and further analyzed with ImageQuant (Amersham Pharmacia Biotech, Sweden). The data were firstly filtered with the following cutoff criteria: the fluorescence intensity of valid spots was set as the average signal intensity of all predefined negative controls plus 3 folds of standard deviation of their signals. The fluorescence signal data which were in line with the above standard in two channels (CY3 and CY5) were accepted as the valid data. The differentially expressed genes were filtered by cy3/cy5 ratios and t-test. The criteria of significant expression change were chosen as the cy $3 /$ cy 5 ratio greater than 2 or less than 0.5 . Subsequently, the genes both in two groups were subtracted from those in HTBS group using Office Excel software (Microsoft, USA), and the left were recognized as the unique genes for HTBS group.

\section{Functional Annotation and Pathway Enrichment Analysis}

Database for Annotation, Visualization and Integrated Discovery (DAVID) [2], which is a web-based tool, provides integrated solutions for the annotation and analysis of genome-scale datasets from microarray. To further infer the functional annotations and enriched pathways of the unique genes for HTBS group, we used DAVID to take analysis of gene enrichment in following functional databases: Gene Ontology (GO), SP-PIR keywords, Kyoto Encyclopedia of Genes and Genomes (KEGG) pathways, REACTOME pathways, and BIOCARTA pathways. The significance level was determined by EASE Score [2], a modified Fisher Exact P-Value and Benjamini-Hochberg False Discovery Rate for testing correction. The threshold of EASE Score was set at 0.1. The genes in the enriched pathways were chosen for further analysis.

\section{J. Protein-Protein Interaction Network Analysis}

To further understand the function of these genes, we used Interologous Interaction Database (I2D) v1.71 (http://ophid.utoronto.ca/i2d) to construct a protein-protein interaction (PPI) network highlighting the interactions between the selected genes for HTBS group [3]. The PPI network was represented as proteins as nodes and interactions between nodes are edges. The PPI network was visualized using Cytoscape (http://www.cytoscape.org/). Hubs were highly connected nodes in a network and vital for the proper function of a network [4]. CytoHubba, a Cytoscape plugin, was used to find the hubs of the PPI network. It evaluated node essentiality by topological characters. The degree of a node was the number of links incident to this node in a network and it was chosen for topological analysis in the study. Top 10 essential nodes ranked by degree scores were selected as hubs from the network. The relationships between the top 10 differentially 
expressed genes were visualized using Cytoscape.

\section{K. RT-PCR verification of Expression Levels}

Quantitative RT-PCR was performed to to assess quantitative gene expression of the differentially expressed specific HTBS genes. Specific PCR primers for the target genes and an invariant housekeeping gene control, ACTG1(actin, gamma 1) were designed using the Primer3 software program, and synthesized by Takara Biotechnology (Dalian) co.,LTD. Subsequently, RT -PCR was performed with the "one-step Prime Script RT-PCR Kit" (Takara Biotechnology, Dalian, China) as described by the manufacturer. Fluorescence detection was performed immediately at the end of each annealing step and the purity of each amplification product was confirmed by generating melting curves. The RT-PCR signal from each gene was normalized by the ACTG1 gene. Expression levels of the genes were measured in terms of threshold cycle value (CT) using the $2^{-\Delta \Delta C t}$ method [5]. The U-Test of Wilcoxon, Mann, and Whitney was applied for estimation of differentially expressed transcripts identified by real-time RT-PCR.

\section{RESULTS}

\section{A. Analysis of Microarray Quality}

The scanned results of the microarray after hybridization (shown in Fig. 1): high intensity of the fluorescence signals, homogeneous background and without obvious defects showed that the quality of the chips was qualified for further microarray analysis. The preliminary evaluation of microarrays' data was determinated by the bivariate analysis between $\mathrm{Cy} 3$ and Cy5 signals of each gene spot on the chips. From the correlated scatter plot of microarray signals (shown in Fig. 2 and Fig. 3), the correlation between the microarray channel readings roughly maintained around $\mathrm{R}=0.9$, which proved that there were no obvious differences between the samples in biological properties.

\section{B. Identification of Differentially Expressed Genes}

There were 174 differentially expressed genes in HTNS group compared with the control group (118 up, 56 down). Compared with the samples in the control group, there were 335 genes in HTBS group whose expression levels had significant difference (174 up, 161 down). After filtered by cy3/cy5 ratios and t-test, a list of 102 genes which both significantly expressed in the two groups were subtracted from those in the HTBS group, and the left 233 genes were regarded as the unique genes for hypertension with blood stasis syndrome.

\section{Identification of Differentially Expressed Genes}

There were 174 differentially expressed genes in HTNS group compared with the control group (118 up, 56 down). Compared with the samples in the control group, there were 335 genes in HTBS group whose expression levels had significant difference (174 up, 161 down). After filtered by cy3/cy5 ratios and t-test, a list of 102 genes which both significantly expressed in the two groups were subtracted from those in the HTBS group, and the left 233 genes were regarded as the unique genes for hypertension with blood stasis syndrome.
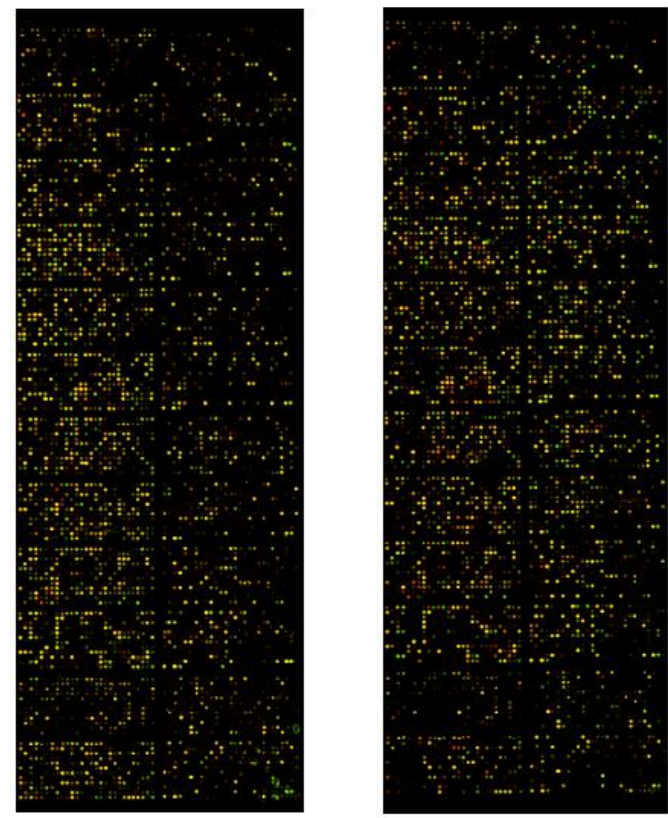

Fig. 1. The scanned results of the microarrays after hybridization. The left is the image of Microarray 2000067478, in which the samples in the HTNS group and corresponding normal reference were labeled by Cy5-dCTP and Cy3- dCTP respectively; and the right is the image of Microarray 2000067479, in which the samples in the HTNS group and corresponding normal reference were labeled by Cy5-dCTP and Cy3- dCTP respectively.

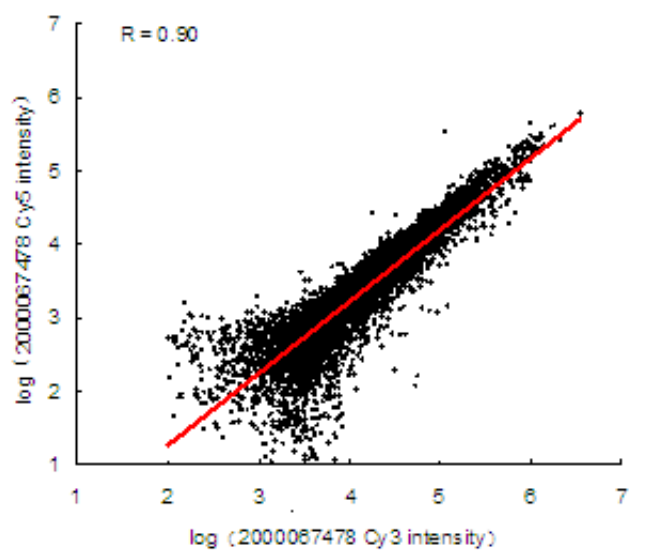

Fig. 2. The correlated scatter plot of microarray signals in Microarray 2000067478

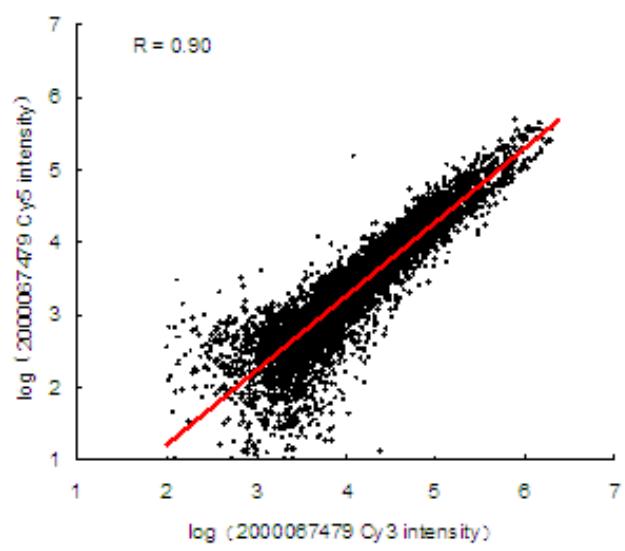

Fig. 3. The correlated scatter plot of microarray signals in Microarray 2000067479.

\section{Analysis of Functional Annotation and Pathway Enrichment}

With the functional annotations analyzed in GO and SPPIR keywords using DAVID, these unique genes for blood stasis syndrome were closely related to enzyme binding, 
cytoskeletal protein binding, protein deacetylase activity, phosphoprotein, hydrolase, nucleotide-binding, disease mutation, atp-binding, transferase, ubl conjugation, endoplasmic reticulum, mitochondrion, etc. The enriched pathways of the unique genes for HTBS group using DAVID. Among the 36 enriched pathways in KEGG, REACTOME and BIOCARTA, cell cycle in mitotic, pathways in cancer, focal adhesion, cytokine-cytokine receptor interaction, MAPK signaling pathway, metabolism of amino acids, HIV Infection, apoptosis and DNA Repair were closely related with blood stasis syndrome. According to the EASE Scores of the enriched pathways, a list of 82 genes was chosen from the unique genes for blood stasis syndrome.

\section{E. Analysis of Protein-Protein Interaction Network}

TABLE I: THE DEgREE SCORES OF THE TOP 10 ESSENTIAL NODES IN THE CONSTRUCTED PPI NETWORK

\begin{tabular}{cccc}
\hline \hline GeneTag & Degree & GeneTag & Degree \\
\hline EGFR & 518 & CDC2 & 196 \\
SRC & 423 & TNFRSF1A & 170 \\
JUN & 350 & STK3 & 155 \\
PCNA & 306 & PSMA1 & 126 \\
CDKN1A & 204 & TCEA1 & 125 \\
\hline \hline
\end{tabular}

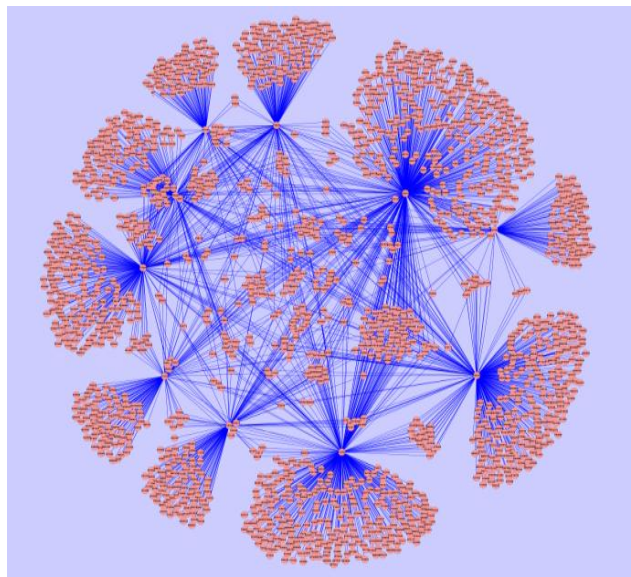

Fig. 4. The PPI network of the selected 82 genes in HTBS group. The hubs of the PPI network were: EGFR, JUN, PCNA, CDKN1A, CDC2, STK3, PSMA1, SRC, TCEA1 and TNFRSF1A.

The chosen 82 genes were imported and analyzed using I2D, and a PPI network with 2892 nodes and 4651 edges was constructed. Based on the network analysis results, the hubs of the PPI network were screened out by CytoHubba. The hubs of the PPI network were as follows: EGFR, JUN, PCNA, CDKN1A, CDC2, STK3, PSMA1, SRC and TNFRSF1A (shown in Table I, Fig. 4), and they were selected as the potential candidate genes for blood stasis syndrome.

\section{F. Verification of Selected Genes by Quantitative Real- Time RT-PCR}

The 10 potential candidate genes for blood stasis syndrome were tested, and the results of the qRT-PCR verification were shown as follows. All the potential candidate genes showed the predicted expression level either higher or lower compared with HT patients with the healthy control $(P<0.05)$. The results of the real-time RT-PCR were consistent with the microarray data (shown in Fig. 5).

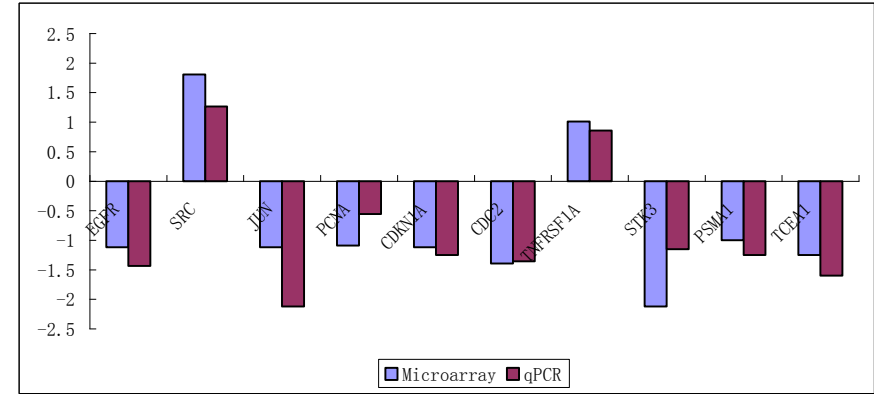

Fig. 5. Comparison between Gene Chip Results and RT-PCR Identification.

\section{CONCLUSION}

As a comprehensive syndrome of TCM, BSS is caused by multiple factors, among which the common pathways are inevitably related to the pathological changes of blood and vessels. As a medium between blood and vessels, the endothelium not only provides a structural barrier between the circulation and surrounding tissue, but ECs also secrete mediators that influence vascular hemodynamics in the physiologic state. Modern medical studies showed that vascular endothelial injury is closely related to the development of blood stasis syndrome [6]. Therefore, the vascular endothelial cells were chosen as the research subjects to study blood stasis syndrome.

Unlike Western medicine, TCM has its unique and integrated theoretical system. TCM holds that the human body is an organic whole in which its constituent parts are inseparable in structure, interrelated and interdependent in physiology, and mutually influential in pathology. It is because TCM's complexity and underlying conceptual foundations that makes lots of researches involved in seeking evidence on whether and how it works. To uncover the underlying molecular mechanisms of BSS, comprehensive analysis of identified genes and their interactions within a network framework might provide many important insights beyond the traditional single-gene or single-marker analyses.

In this research, microarray data mining and network analysis were integrated to examine the potential candidate genes for hypertension with blood stasis syndrome. Compared with previous methodologies reported in the literatures, the proposed microarray and data mining method has shown a great improvement in analyzing BSS. Microarray data mining and network analysis can provide a basis for investigating the molecular mechanisms of BSS, identify potential therapeutic targets, and facilitate the development of traditional Chinese medicine-derived biopharmaceutical products.

\section{REFERENCES}

[1] Y. N. Song, P. J. Biao, X. Li et al., "Effect of liangxuehuayu recipe on hemorheology in rats with blood stasis syndrome," Asian Pacific Journal of Tropical Medicine, vol. 5, pp. 935-938, Dec. 2012.

[2] G. Jr. Dennis, B. T. Sherman, D. A. Hosack et al., "DAVID: Database for annotation, visualization, and integrated discovery," Genome Biology, vol. 4, pp. P3, May 2003.

[3] B. M. Gordon, J. Igor, Y. Yosef et al., "Genomic amplicons target vesicle recycling in breast cancer," J Clin Invest., vol. 119, pp. 21232127, Aug. 2009.

[4] J. Wang and G. Yu, "A systems biology approach to characterize biomarkers for blood stasis syndrome of unstable angina patients by integrating microrna and messenger rna expression profiling," $J$ 
Evidence-Based Complementary and Alternative Medicine, vol. 2013, 2013.

[5] Q. Luo, Y. H. Lian, J. Q. Su et al., "Effect of modified sijunzi decoction on 5-ht receptors in intestinal m ucosa of ibs-d patients with spleen-dificiency syndrome," Chinese Journal of Information on Traditional Chinese Medicine, vol. 20, pp. 19-21, Jan. 2013.

[6] Y. H. Zhou, L. G. Chen, and Y. Qu, "Studies on the vascular endothelial cell injury model of blood stasis syndrome from the angle of combination of disease identification and syndrome differentiation," Chinese Journal of Integrated Chinese and Western Medicine., vol. 31, pp. 696-700, May 2011.

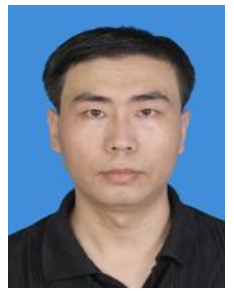

Lian Yong-Hong was graduated from Guangzhou University of Chinese Medicine. He got his P.H.D degree of Chinese Medicine in July, 2012. His specialized field is: bioinformatics for Chinese Medicine. He is now in the Institute of Integrated Chinese and Western Medicine, Jinan University, Guangzhou, China.
Li Wen-Juan was graduated from Jinan University. She got her master degree of pathophysiology in July, 2008. She is now in the Medical school of Jinan University, Guangzhou, China.

Chen Li-Guo is a professor and a doctoral adviser. He graduated from Shandong University of Traditional Chinese Medicine, he got his P.H.D degree of Chinese Medicine in July, 1990. His specialized field is: the mechanism of TCM zheng. He is now in the Institute of Integrated Chinese and Western Medicine, Jinan University, Guangzhou, China. 